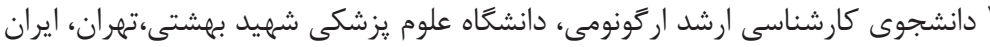

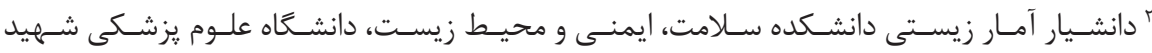

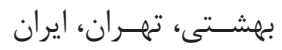

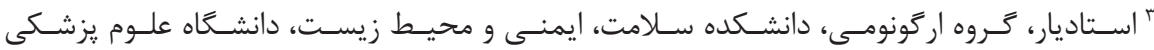

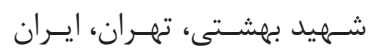

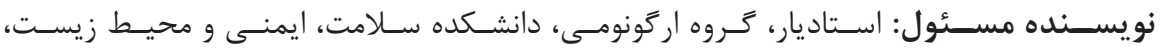

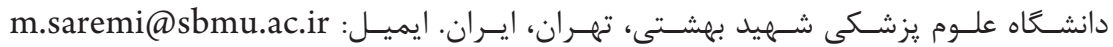

DOI: $10.21859 /$ joe- 0402416

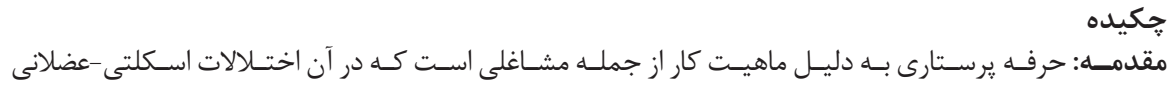

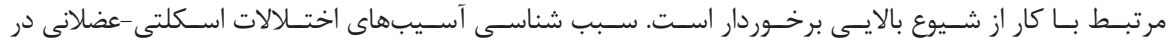

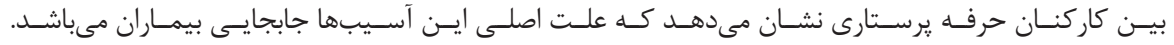

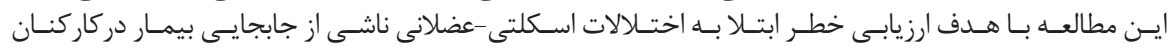

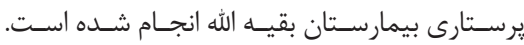

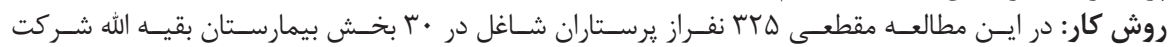

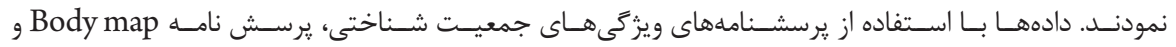

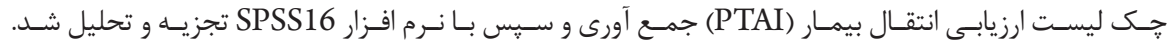

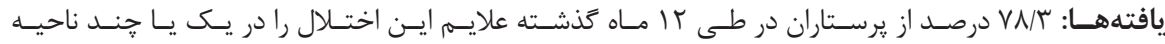

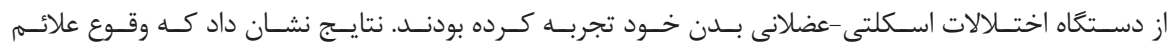

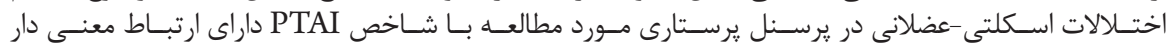
تاريخ دريافت مقاله: | || ||

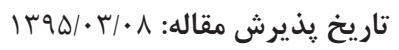

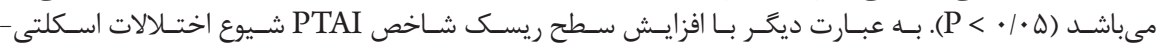

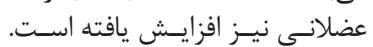

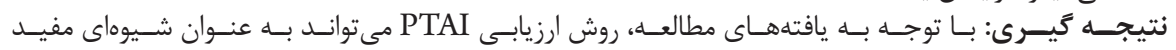

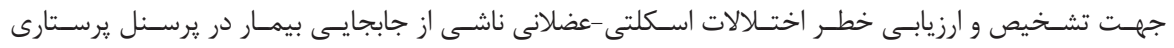

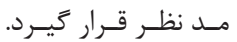

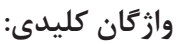
اختلالات اسكلتى -عضلانى كليدى يرستار جابجايى بيمار شاخص PTAI

تمامى حقوق نشر براى دانشكاه علوم يزشكى همدان محفوظ است.

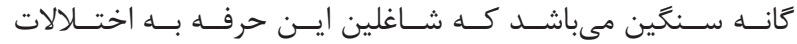

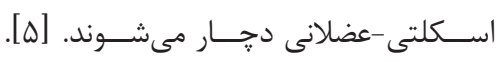

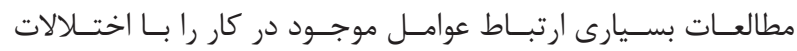

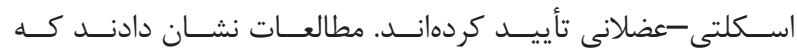

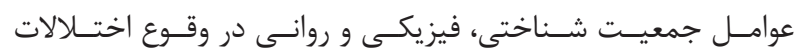

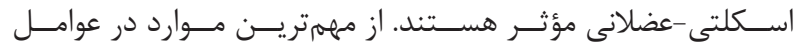

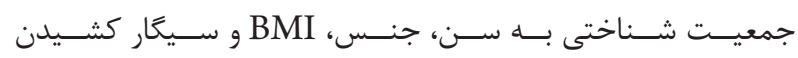

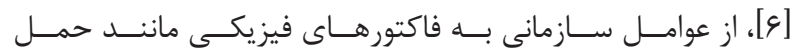

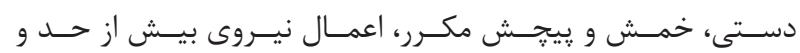

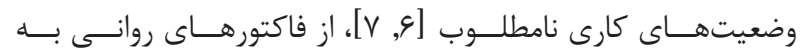

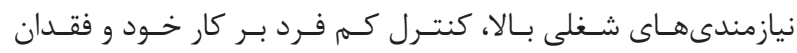

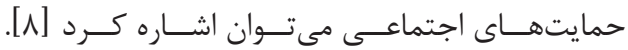

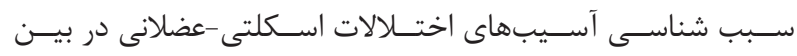

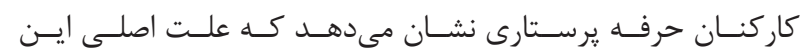

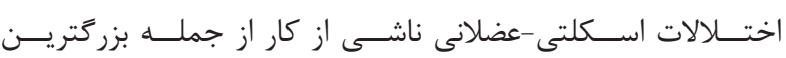

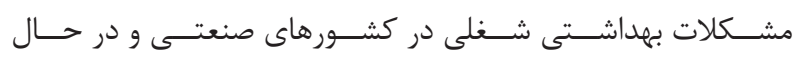

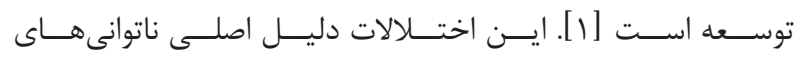

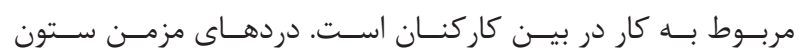

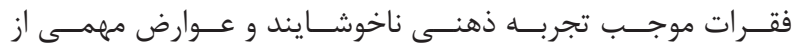

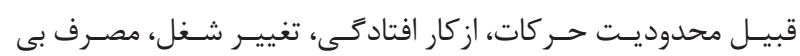

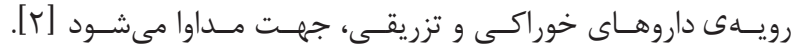

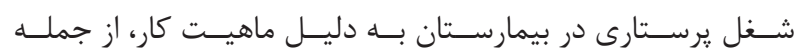

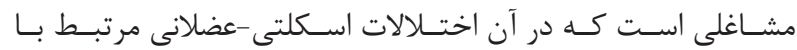

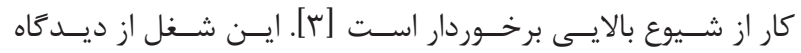

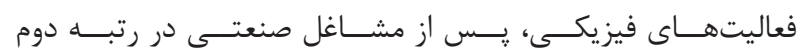

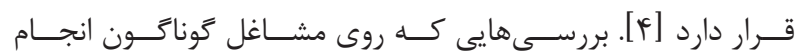

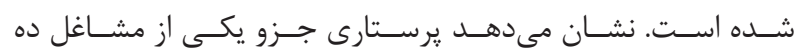


كاركنـان برسـتارى منجــر بــه كاهـش كيفيـت خدمــات يرسـتارى

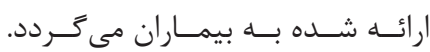

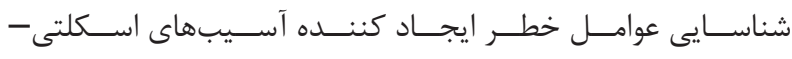

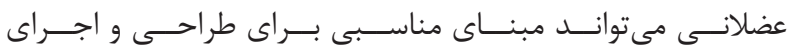

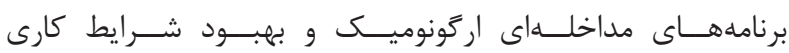

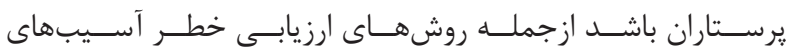

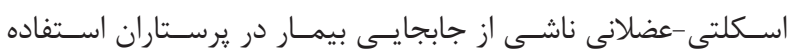

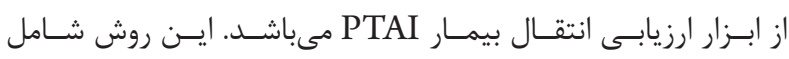

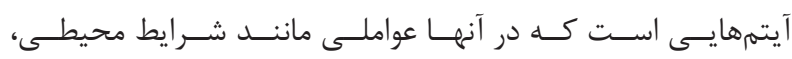

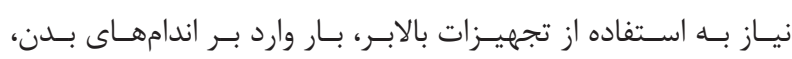

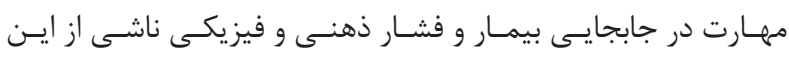

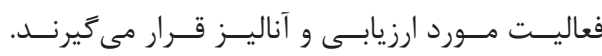

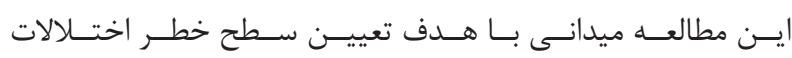

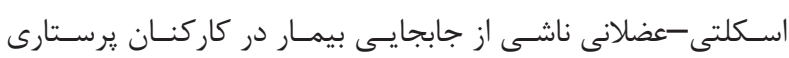

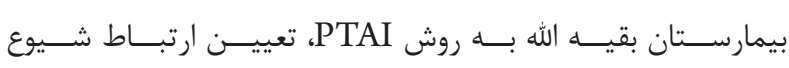

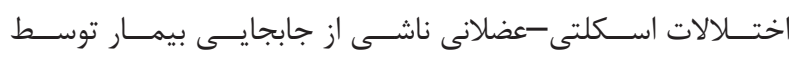

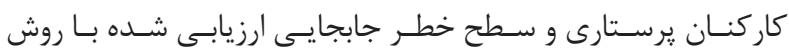

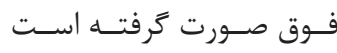

روش كار

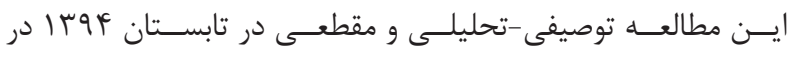

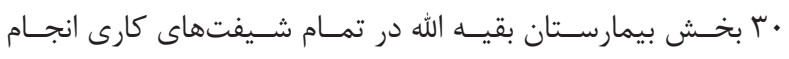

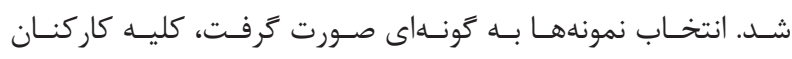
يرسـتارى بخشهــاى بسـترى بيمارسـتان كـهـ در جابجايـى بيمـار

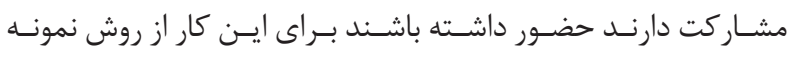

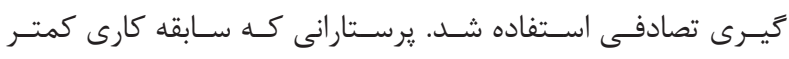

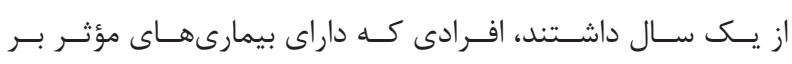

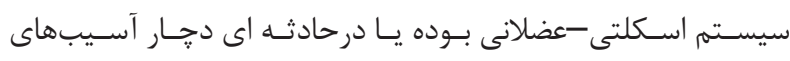

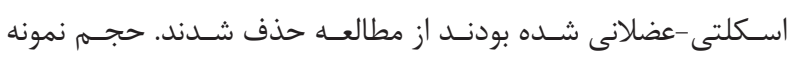

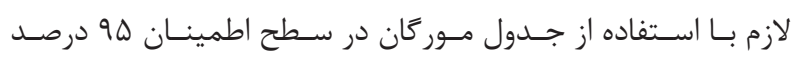

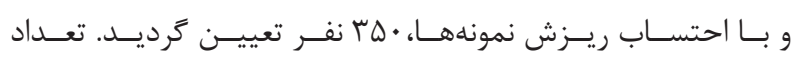

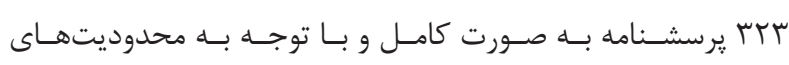

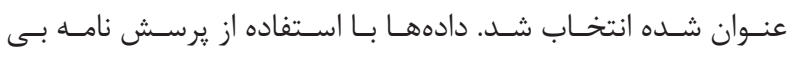

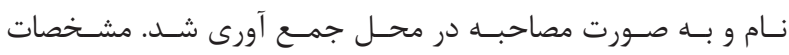

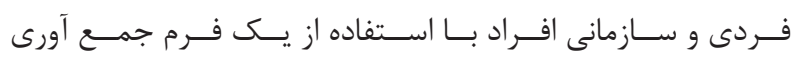

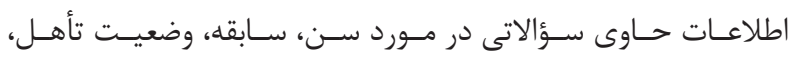

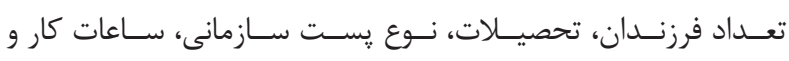

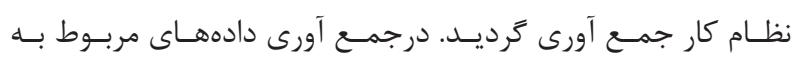

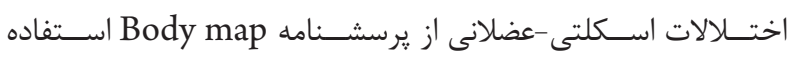

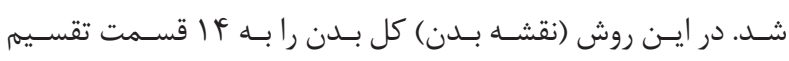

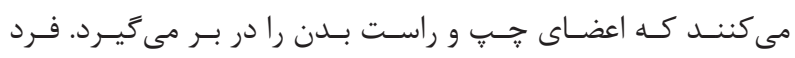

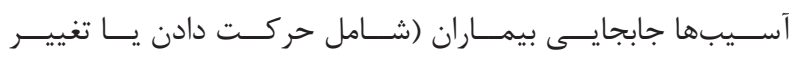

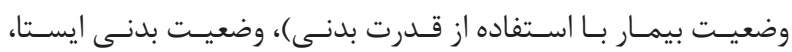

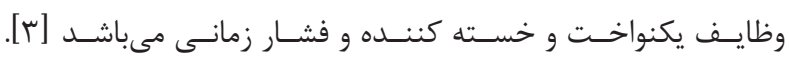

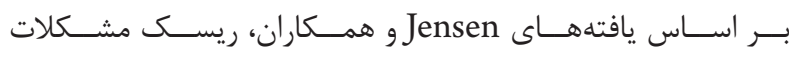

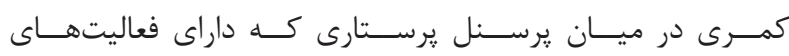

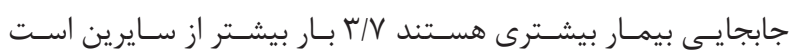

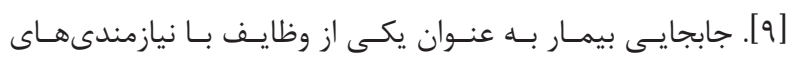

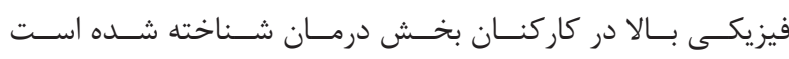

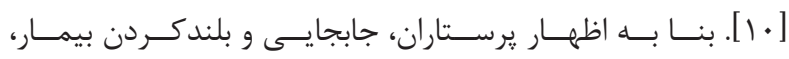

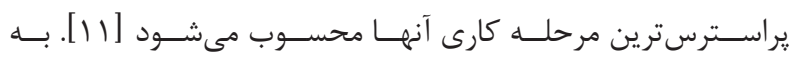

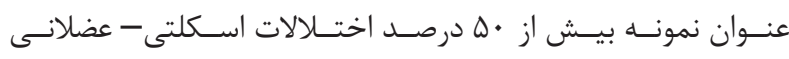

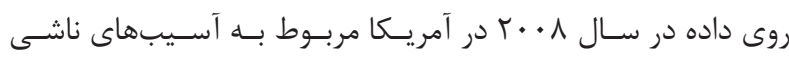

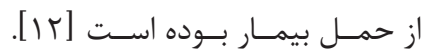

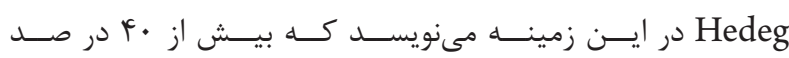

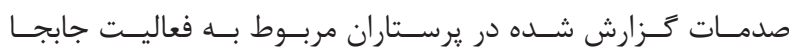

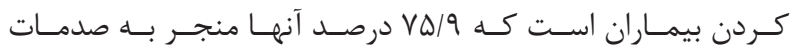

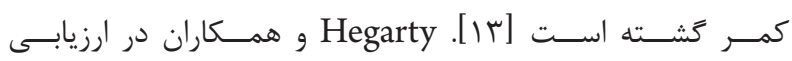

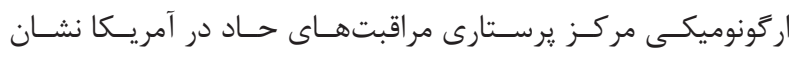

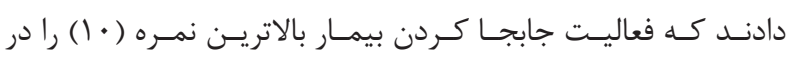

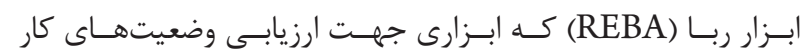

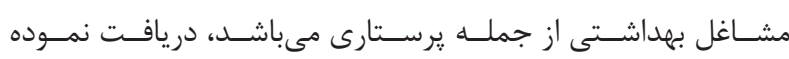

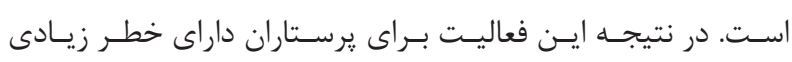

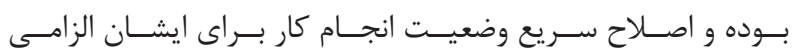

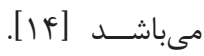
در مطالعـهاى كــه توســط آليســون و همــكاران خــزارش شـــه،

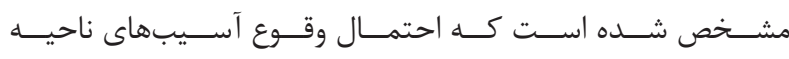

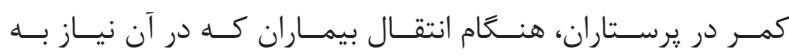

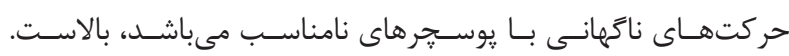

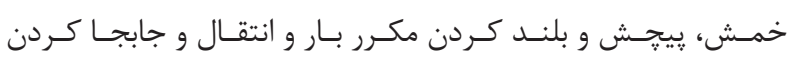

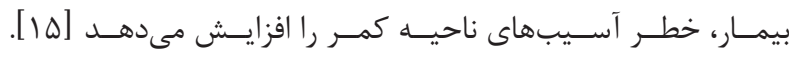

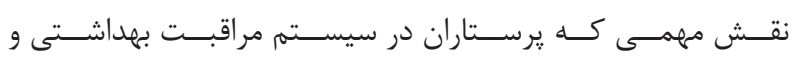

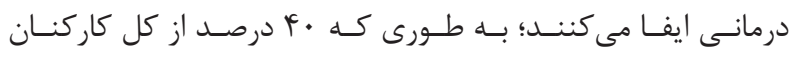

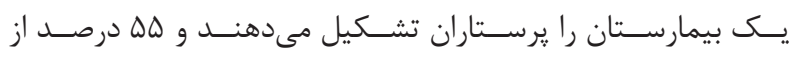

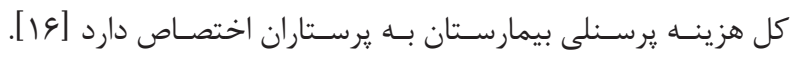

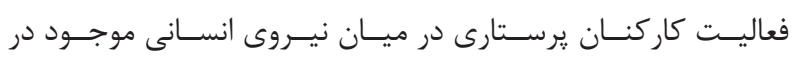

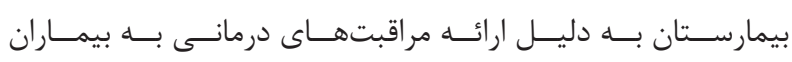

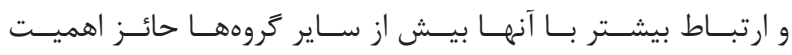

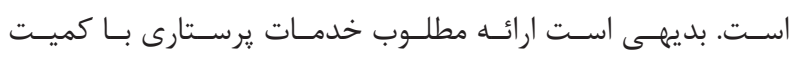

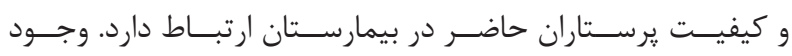

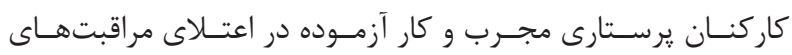

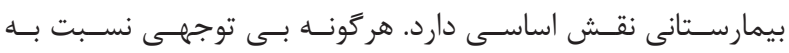




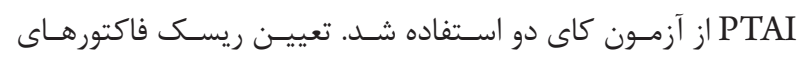

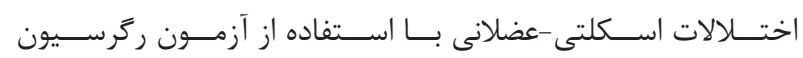
لجسـتيك انجـام شـد.

\section{يافتهها}

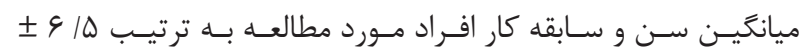

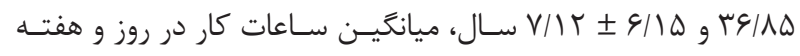

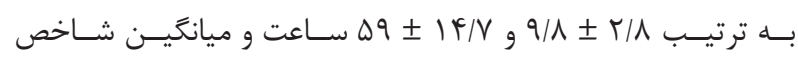
(

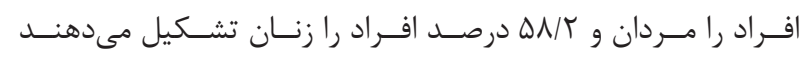
ز

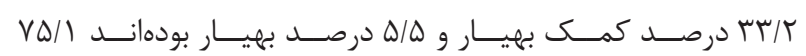

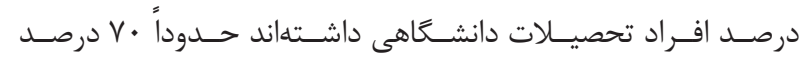

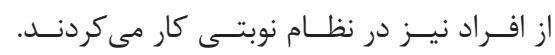

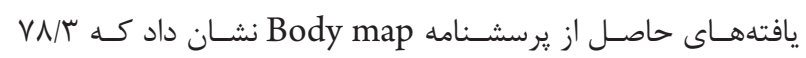

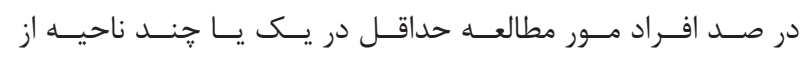

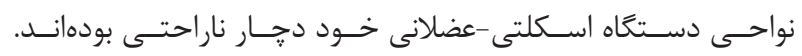

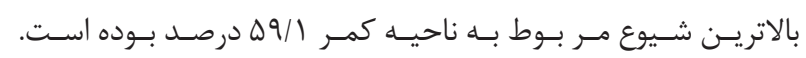

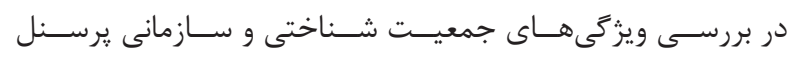

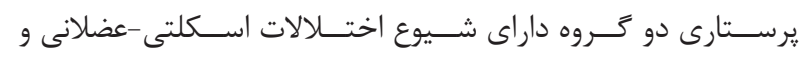

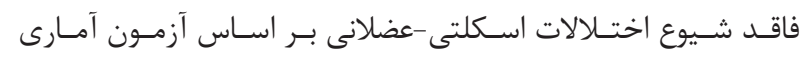

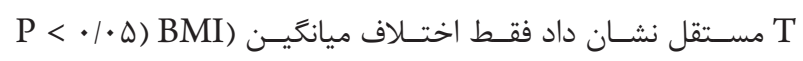

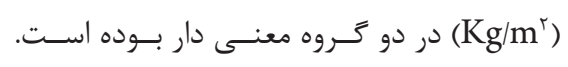

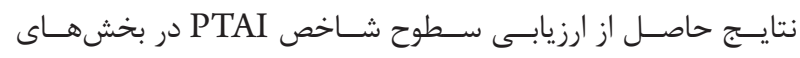

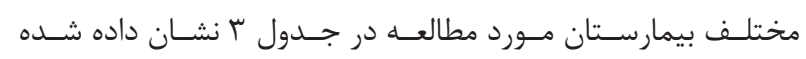

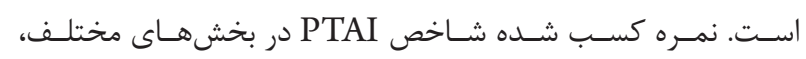

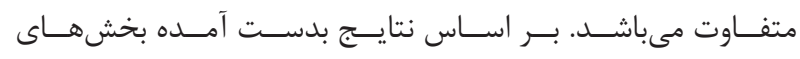

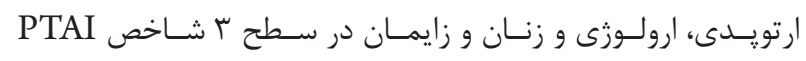

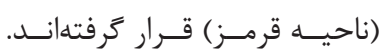

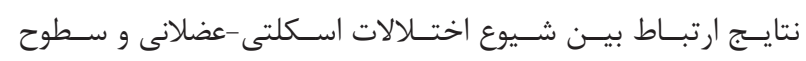

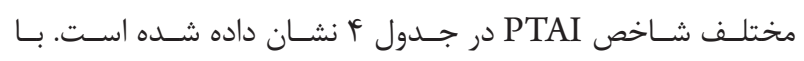

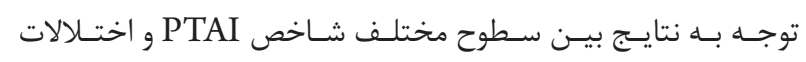

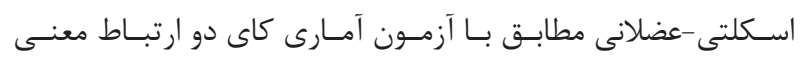

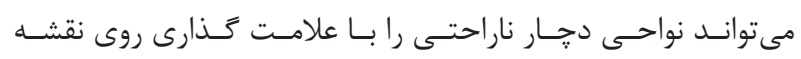

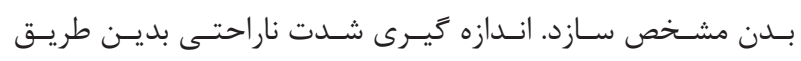

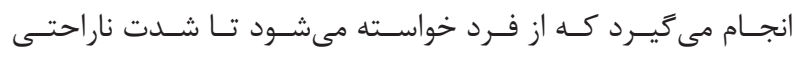

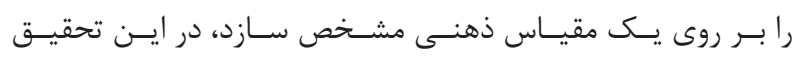

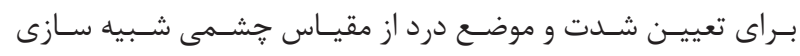

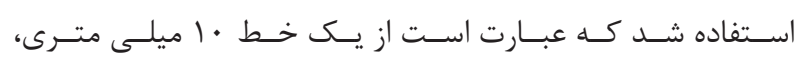

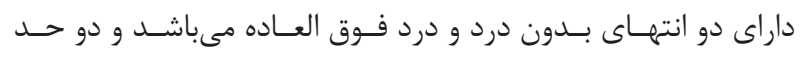

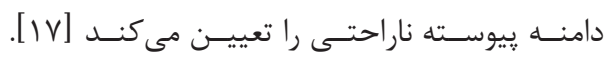

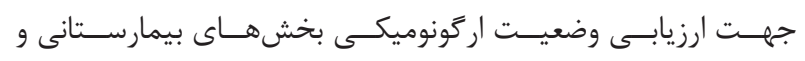

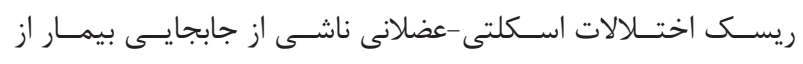

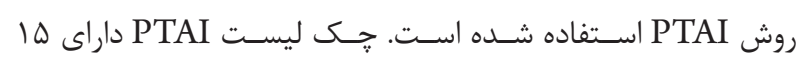

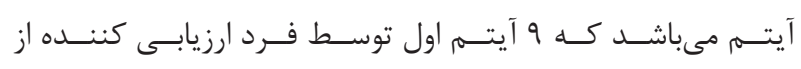

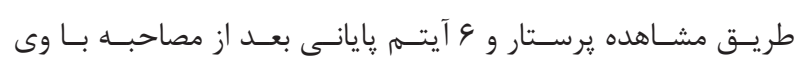

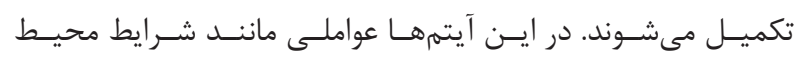

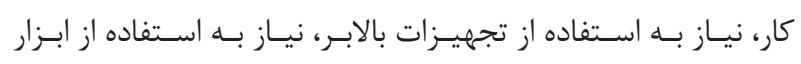

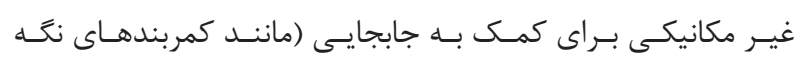

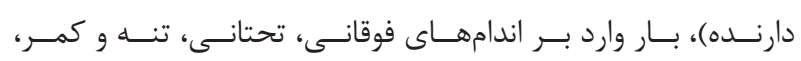

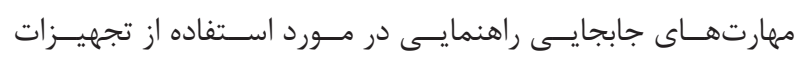

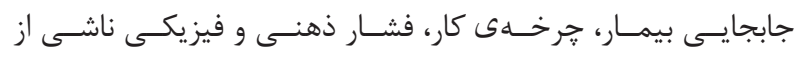

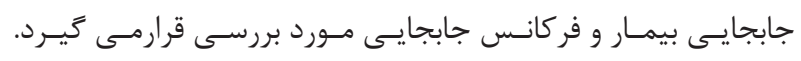

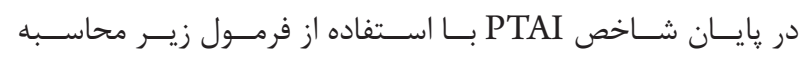

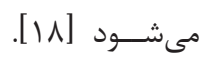

$$
\frac{a+(0.67 b)+(0.33 c)}{d} \times 100=\%
$$

a : تعداد آيتمهاى صحيح : a

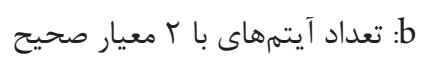

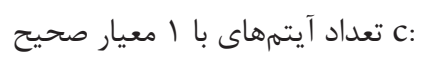

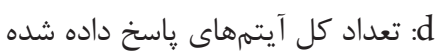

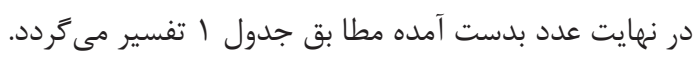

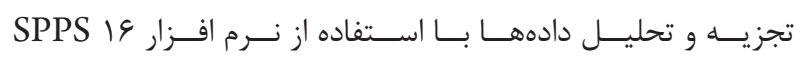

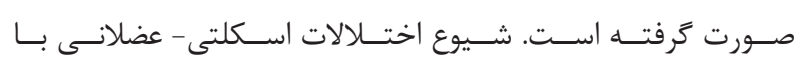

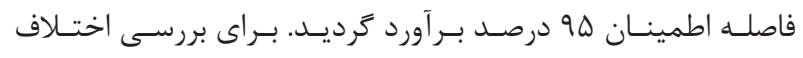

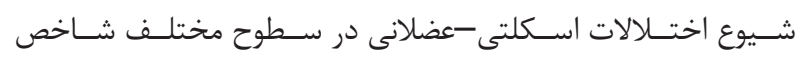

\begin{tabular}{|c|c|c|}
\hline \multicolumn{3}{|c|}{ جدول ا: سطوح خطر بر اساس شاخص PTAI با توجه به امتياز محاسبه شده } \\
\hline سطح & ناحيه & متياز PTAI \\
\hline 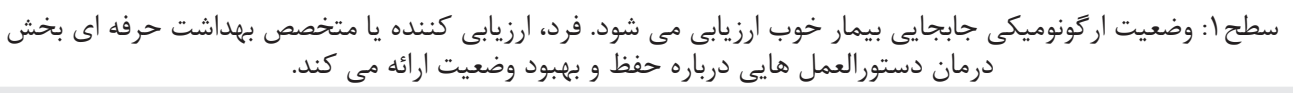 & سبز & $\% \wedge \cdot<$ \\
\hline سطح ז: فشار ناشى از جابجايى بيمار بالا و بايد اقداماتى در جهت اصلاح، مشكلات شناسايى شده در فرم ارزيابى در محيط & زرد & $\% \varphi+-\% \Lambda$. \\
\hline 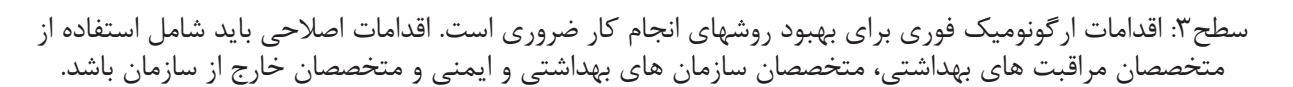 & 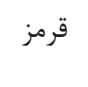 & $<\%$ \\
\hline
\end{tabular}




\begin{tabular}{|c|c|c|c|c|c|c|c|}
\hline \multirow{4}{*}{ آزمون آمارى } & \multicolumn{7}{|c|}{ جدول r: بررسى وابستخى اختلالات اسكلتى عضلانى و ويزُخى هاى جمعيت شناختى و سازمانى } \\
\hline & \multicolumn{4}{|c|}{ اختلالات اسكلتى عضلانى } & \multicolumn{2}{|l|}{ كل } & \multirow[t]{3}{*}{ كروه متغير } \\
\hline & \multicolumn{2}{|c|}{ ندارد (n= (n) } & \multicolumn{2}{|c|}{ دارد (n= (n) (253) } & \multirow[b]{2}{*}{ انحراف استاندارد } & \multirow[b]{2}{*}{ ميانگين } & \\
\hline & انحراف استاندارد & ميانگين & انحراف استاندارد & ميانكين & & & \\
\hline$\cdot / 4 \cdot 1$ & N/qV & re/ra & $N / \Delta$ & rg/AD & N/9 & re/V & سن (سال) \\
\hline$\cdot / \cdot r \Delta$ & $r / 9$ & $r F / 9$ & $\Gamma / \Lambda$ & $r \Delta / V$ & $\mathrm{r} / \Lambda$ & $r \Delta / \Delta$ & BMI $\left(\mathbf{k g} / \mathbf{m}^{2}\right)$ \\
\hline .1119 & $r / \Delta$ & $9 / \mu$ & $r / \Lambda$ & $9 / 1$ & T/VT & $9 / \mathrm{V}$ & ساعت كار در روز(ساعت) \\
\hline$\cdot /$ ATV & 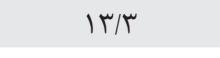 & $\Delta V$ & $\mid F / V$ & 09 & $\mid F / V$ & $\Delta q / 1 r$ & ساعت كار در هفته(ساعت) \\
\hline \multirow[t]{2}{*}{ ( ) } & $\Delta / \mathcal{F}$ & $\varepsilon / 4$ & $9 / 10$ & V/IT & $9 / 10$ & $1 T / 9$ & سابقه كار (سال) \\
\hline & & & & & & & جنسيت \\
\hline \multirow[t]{3}{*}{$\cdot / l V$} & $\Delta F / T$ & rᄉ & rN/r & qV & $\Delta \Lambda / T$ & 111 & زن - ان \\
\hline & $f \Delta / V$ & rt & $91 / V$ & 109 & $f \mid / \Lambda$ & Ird & 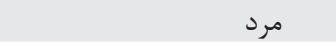 \\
\hline & & & & & & & وضعيت تأهل \\
\hline \multirow[t]{3}{*}{$\cdot 1 \cdot 19$} & $r V / I$ & 19 & $r \Delta / r$ & gr & $r \Delta / V$ & $\Lambda$ & مجرد \\
\hline & $V T / 9$ & DI & $V F / V$ & 119 & $V \Psi / r$ & TF. & متاهل \\
\hline & & & & & & & تحصيلات \\
\hline \multirow[t]{4}{*}{ ( ) } & $T F / T$ & IV & $r F / q$ & st & $r F / \Lambda$ & $\wedge \cdot$ & زير دييلم و ديِلم \\
\hline & $10 / V$ & 11 & $\mid r / 4$ & re & $14 / 9$ & $r \Delta$ & فوق دييلم \\
\hline & 4. & fr & $91 / V$ & 109 & $91 / 4$ & 191 & ليسانس و بالاتر \\
\hline & & & & & & & شغل \\
\hline \multirow[t]{4}{*}{ - MFY } & r. & th & Tr/T & $\lambda F$ & $r T / \Delta$ & $1 \cdot 0$ & كمك بهيار \\
\hline & $\mathrm{V} / \mathrm{I}$ & $\Delta$ & Q/1 & Ir & $\Delta / 9$ & 11 & بهيار \\
\hline & sT/9 & fy & 4)/V & 109 & $91 / 9$ & $r \cdot$. & يرستار \\
\hline & & & & & & & نظام كار \\
\hline \multirow[t]{2}{*}{1} & $f \mid / d$ & $r 9$ & $r \cdot / l$ & VG & $r q$ & 94 & ثابت كار \\
\hline & $\Delta N / \varphi$ & (i) & $99 / 9$ & IVV & VI & rrq & نوبت كار \\
\hline
\end{tabular}

\begin{tabular}{|c|c|c|c|}
\hline \multirow[b]{2}{*}{ 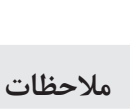 } & \multicolumn{3}{|c|}{ جدول rّ: ارزيابى سطح شاخص PTAI در بخشهاى بيمارستان مورد مطالعه } \\
\hline & سطح & 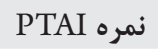 & بخش \\
\hline 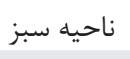 & 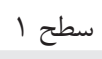 & $\wedge \Delta$ & اطفال \\
\hline ناحيه زرد & 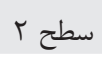 & ve & 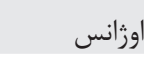 \\
\hline ناحيه زرد & سطح r & $V \Delta / \Delta$ & ICU \\
\hline ن احيه زرد & 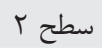 & $\checkmark \Delta$ & جراحى \\
\hline ن احيه زرد & 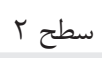 & ve & $\mathrm{CCU}$ \\
\hline ن احيه زرد & 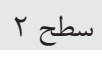 & ve & 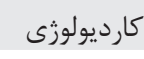 \\
\hline ن احيه زرد & سطح & Vr & 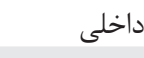 \\
\hline 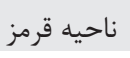 & 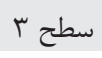 & 99 & 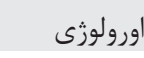 \\
\hline 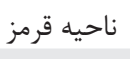 & 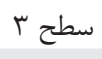 & 99 & 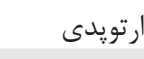 \\
\hline 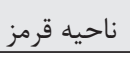 & 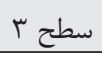 & $\Delta V / \Delta$ & زنان و زايمان \\
\hline
\end{tabular}




\begin{tabular}{|c|c|c|c|c|c|c|}
\hline \multirow{4}{*}{ P-Value } & \multicolumn{6}{|c|}{ جدA جأ: ارتباط شيوع اختلالات اسكلتى-عضلانى و سطوح مختلف شاخص PTAI } \\
\hline & \multicolumn{4}{|c|}{ اختلالات اسكلتى عضلانى } & تعداد & سطح شاخص PTAI \\
\hline & \multicolumn{2}{|c|}{ ن ندارد } & \multicolumn{2}{|c|}{ دارد } & & \\
\hline & 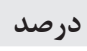 & 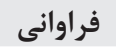 & 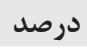 & 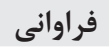 & & \\
\hline \multirow{3}{*}{. } & $i v / V$ & re & $\Delta T / T$ & 11 & fe & 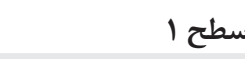 \\
\hline & $I V / V$ & rt & $\Lambda r / r$ & 149 & $|\wedge|$ & 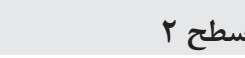 \\
\hline & $19 / 0$ & 19 & $\Lambda \mu / \Delta$ & 11 & qV & سطح r \\
\hline
\end{tabular}

\begin{tabular}{|c|c|c|c|}
\hline \multirow[b]{2}{*}{ مقدار احتمال } & \multicolumn{3}{|c|}{ جدول ه: نتايج آناليز ركر سيون جهت بررسى ميزان نسبت بخت تعديل شده اختلالات اسكلتى-عضلانى } \\
\hline & فاصله اطمينان & نسبت بخت تعديل شده OR & متغير \\
\hline & & & 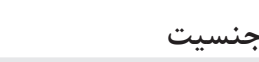 \\
\hline & & 1 & مرد \\
\hline $\mathrm{V} \mathrm{V} / \cdot$ & $r \Delta / r-r) / \cdot$ & $1 / \cdot 4$ & زن \\
\hline $\mid \omega / \cdot$ & & $r /$. & BMI \\
\hline $\mid \kappa / \cdot$ & $4 / \pi-\cdot 9 / 1$ & $1 / 14$ & سابقه كار(سال) \\
\hline \multirow[t]{3}{*}{$|N| \cdot$} & $r T / T-\cdot T / l$ & $1 / 11$ & ساعت كار در هفته \\
\hline & & & 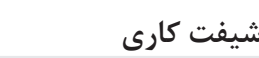 \\
\hline & & 1 & روزكار \\
\hline \multirow[t]{3}{*}{$|\& N| \cdot$} & $1-1 / \pi 9$ & $r \Delta / 1$ & شيفت كار \\
\hline & & & شغل \\
\hline & & 1 & يرستار \\
\hline \multirow[t]{3}{*}{$\cdot / \pi 9$} & $1 / \cdot r-r / r V$ & $\cdot \Delta / 1$ & كمك بهيار \\
\hline & & & شاخص PTAI \\
\hline & & 1 & سطح 1 \\
\hline$\cdot 1 \cdot \Delta$ & $1 / T \Lambda-I F / A F$ & FT/T & سطح r \\
\hline .1 .1 & $1 / 9 V-11 / r r$ & $V V / F$ & سطح \\
\hline
\end{tabular}

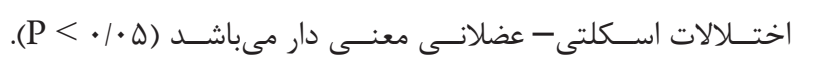

\section{بحث}

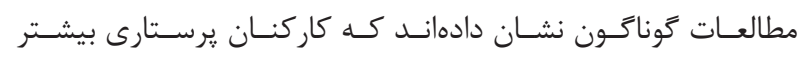

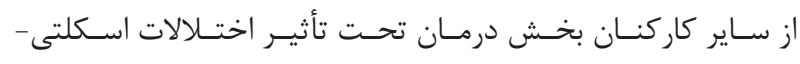

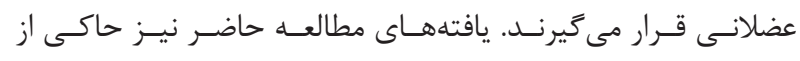

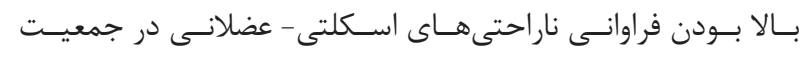

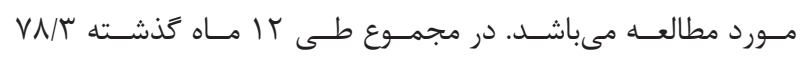

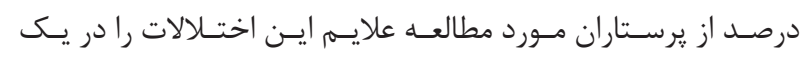

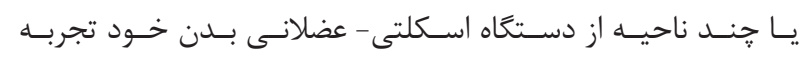

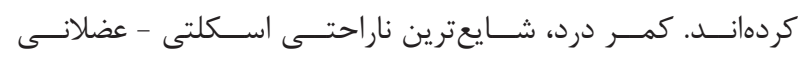

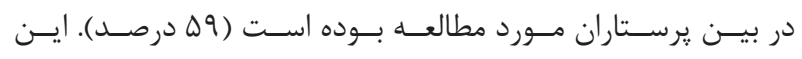

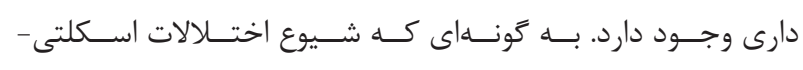

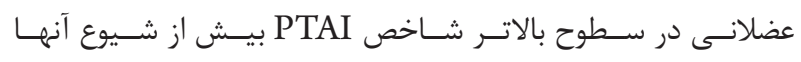

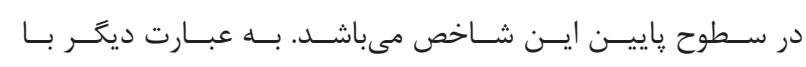

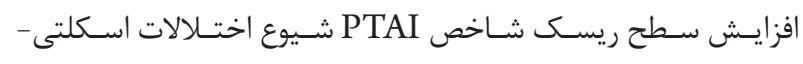

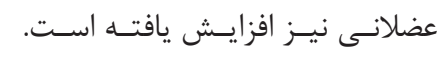

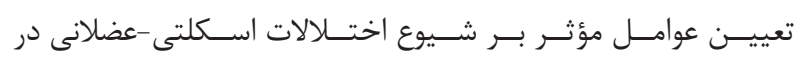

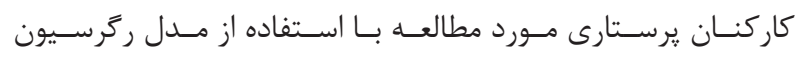

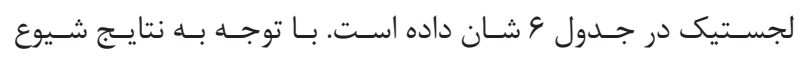

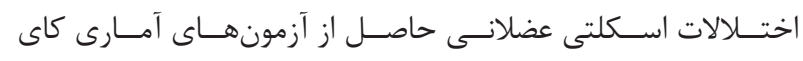

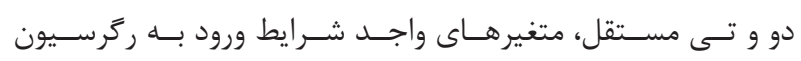

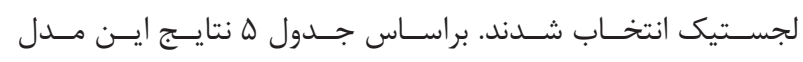

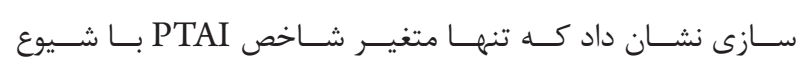


انجـام گرفـت نتايسج بدست آمسده نشـان دهنـده آن بـود

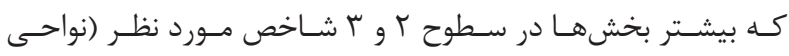

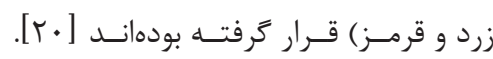

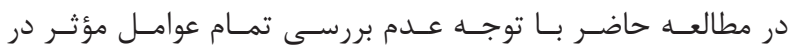

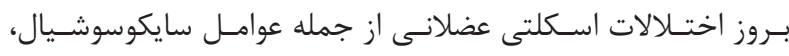

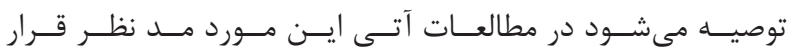

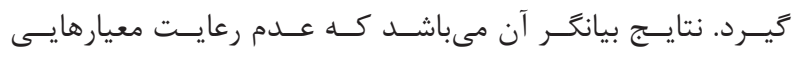

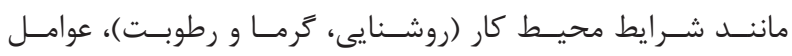

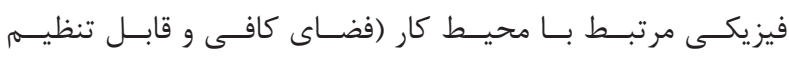

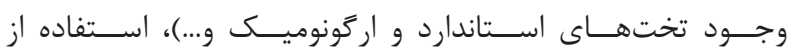

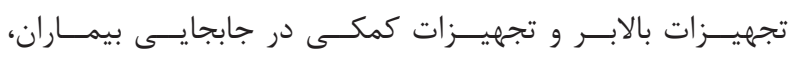

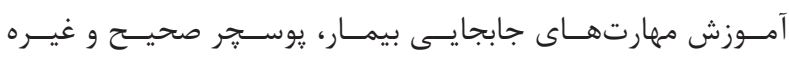

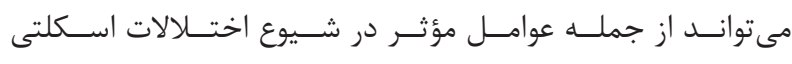

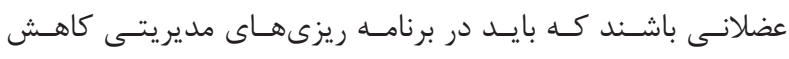

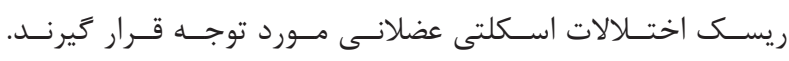

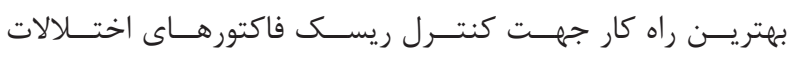

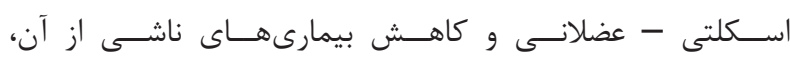
رويكردهــاى كنتر لـى مهندسـى و كنتــر لـى مديريتـى مى باشـــد

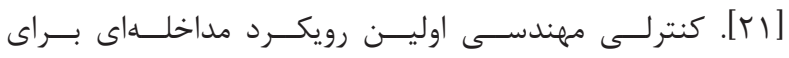

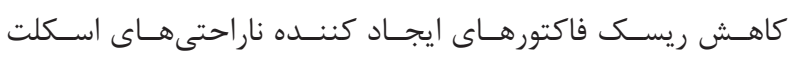

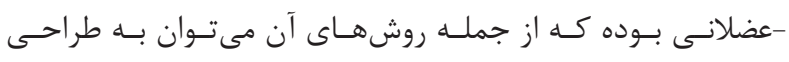

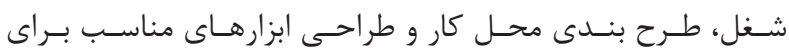

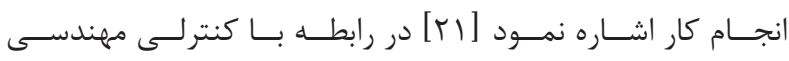

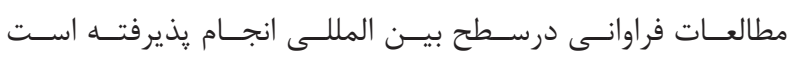

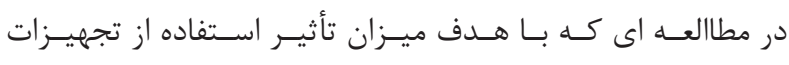

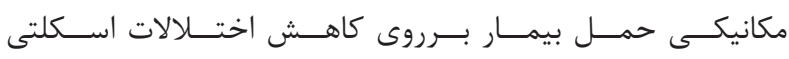

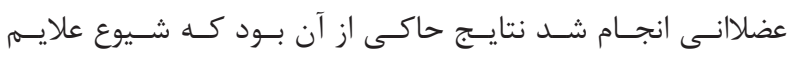

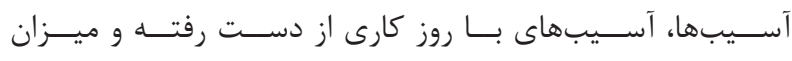

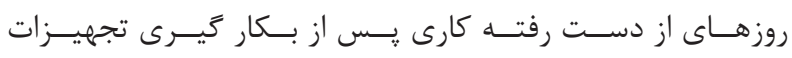

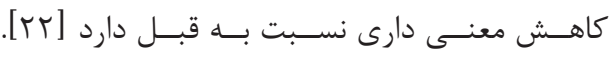

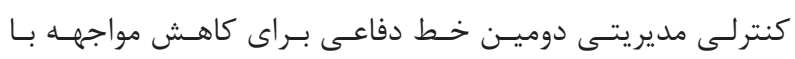

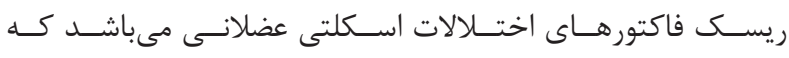

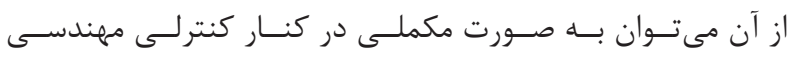

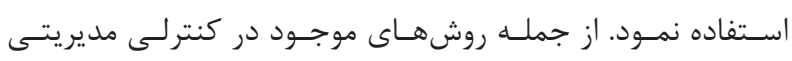

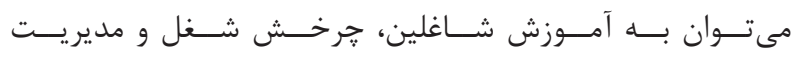

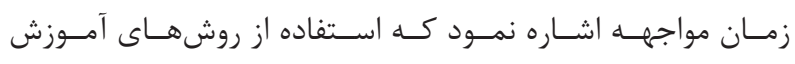

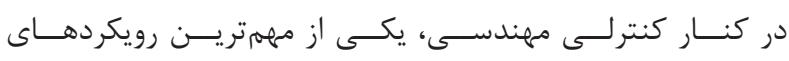

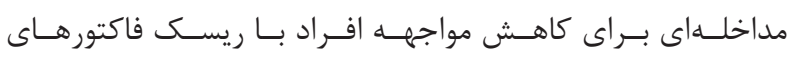

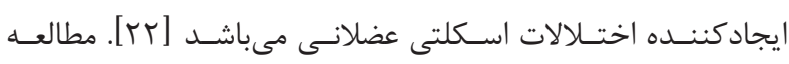

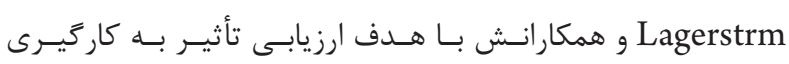

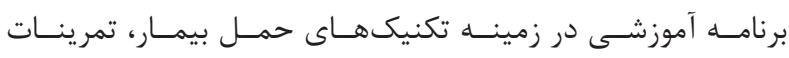

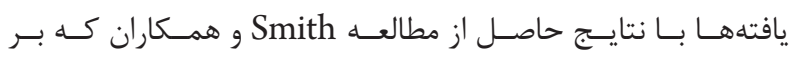

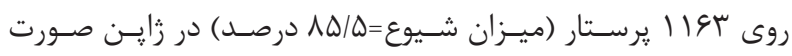

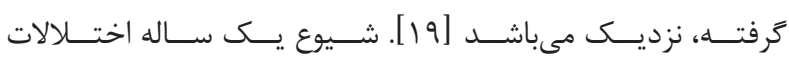

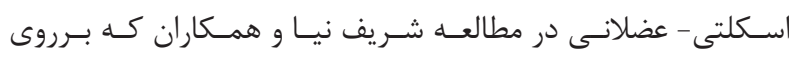

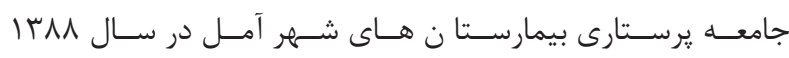

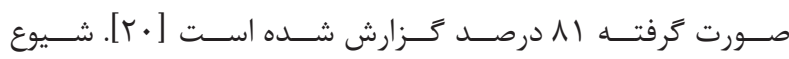
اختـلالات اسـكلتى - عضلانسى در مطالعـه جوبينـهـ و همـكاران كـهـ بر

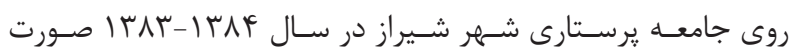

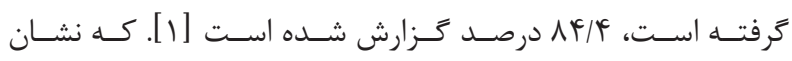

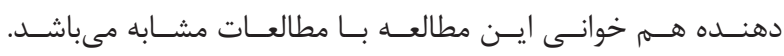

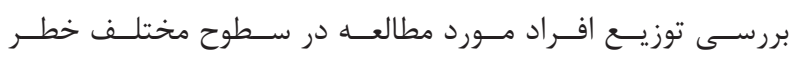

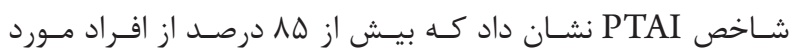

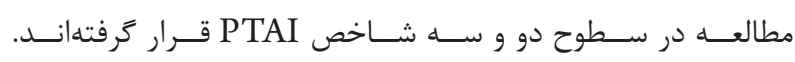

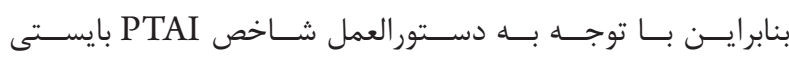

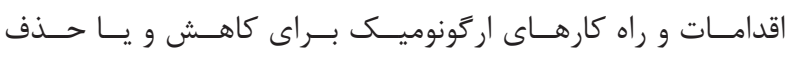

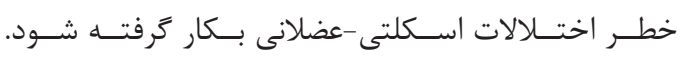

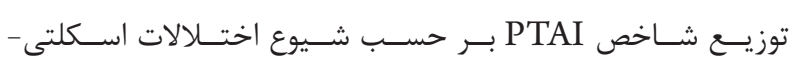

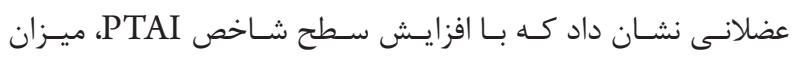

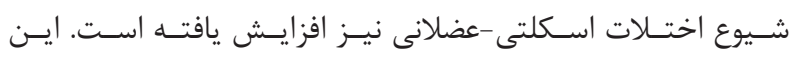

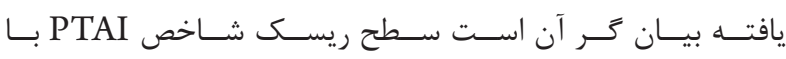

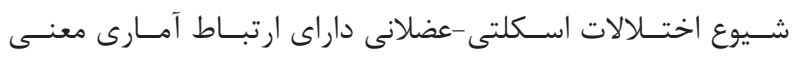

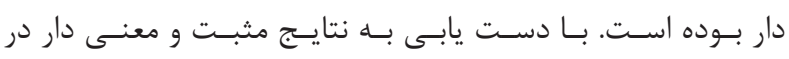

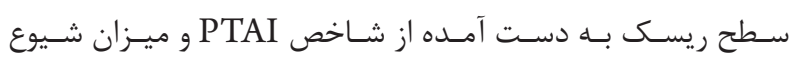

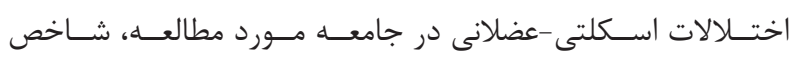

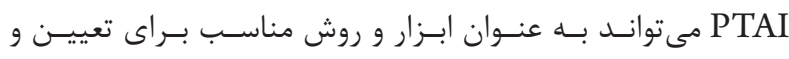

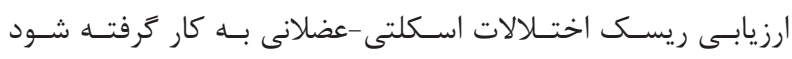

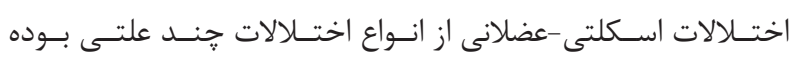

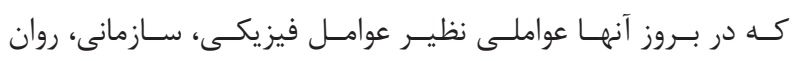

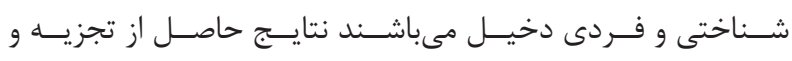

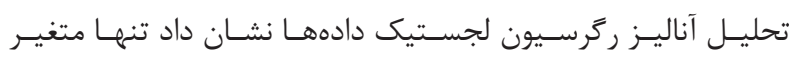
شـاخص PTAI بـا شـيوع اختـلالات اسـكلتى -عضلانى معنسى دار

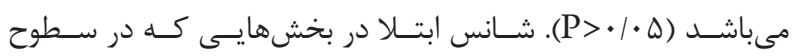

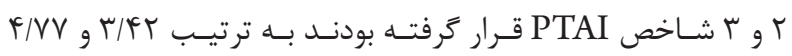

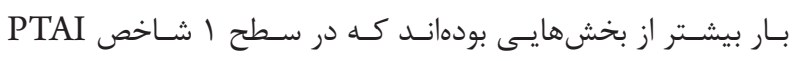

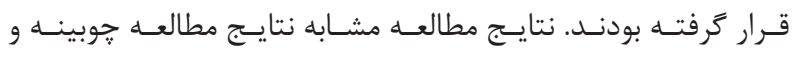

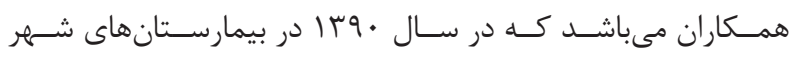

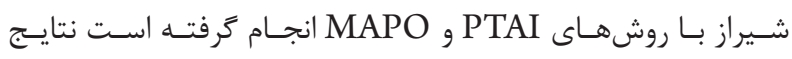

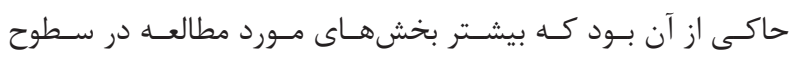

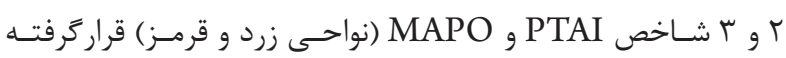

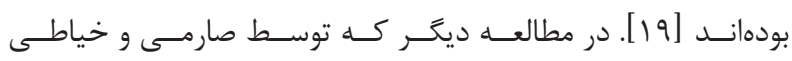

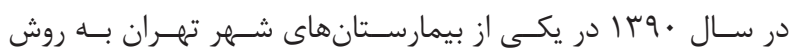




$$
\begin{aligned}
& \text { اختـالات اسـكلتى -عضلانى ناشـى از جابجايـى بيمــار در يرســل } \\
& \text { يرسـتارى مـد نظــر قـرار حيــرد. } \\
& \text { سبإسكز الوى }
\end{aligned}
$$

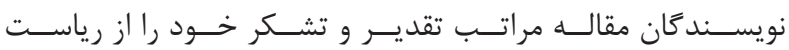

$$
\begin{aligned}
& \text { محتــرم بيمارسـتان، معاونـت محتــرم آموزشـى، مديريـت محتــرم }
\end{aligned}
$$

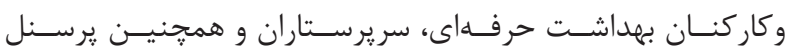

$$
\begin{aligned}
& \text { يرســتارى كـــه در ايــن تحقيــق همـــارى نمودهانــد، اعــلام } \\
& \text { مى نما ينـــد. }
\end{aligned}
$$

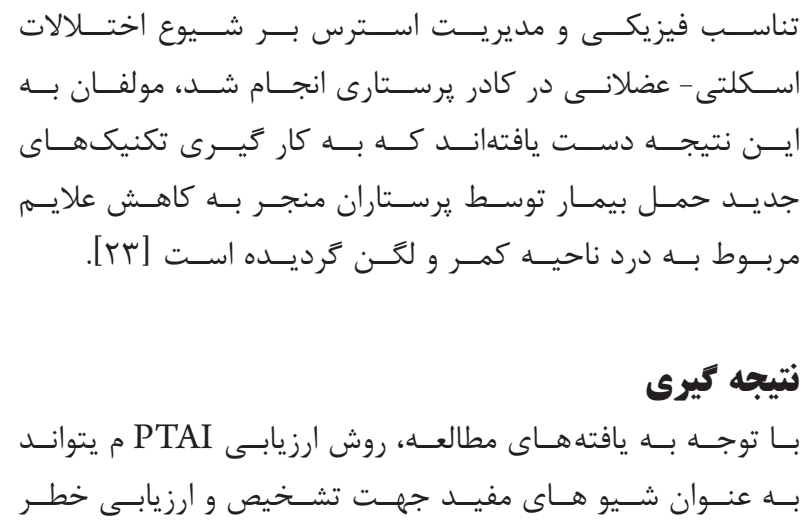

\section{REFERENCES}

1. Choobineh A, Rajaeefard AR, Neghab M. [Perceived demands and musculoskeletal disorders among hospital nurses]. Hakim 2007;2.

2. Balague F, Mannion AF, Pellise F. Clinical update: Low back pain: Lancet 2007.

3. Menzel NN, Brooks SM, Bernard TE ,Nelson A. The physical workload of nursing personnel: association with musculoskeletal discomfort. Int J Nurs Stud. 2004;41(8):859-67. DOI: 10.1016/j. ijnurstu.2004.03.012 PMID: 15476759

4. Engels JA, van der Gulden JW, Senden TF, van't Hof B. Work related risk factors for musculoskeletal complaints in the nursing profession: results of a questionnaire survey. Occup Environ Med. 1996;53(9):636-41. PMID: 8882121

5. Smith DR, Ohmura K, Yamagata Z, Minai J. Musculoskeletal disorders among female nurses in a rural Japanese hospital. Nurs Health Sci. 2003;5(3):185-8. PMID: 12877719

6. Burdorf A, Sorock G. Positive and negative evidence of risk factors for back disorders. Scand J Work Environ Health. 1997;23(4):243-56. PMID: 9322815

7. Hales TR, Bernard BP. Epidemiology of work-related musculoskeletal disorders. Orthop Clin North Am. 1996;27(4):679-709. PMID: $\underline{8823390}$

8. Bongers PM, de Winter CR, Kompier MA, Hildebrandt VH. Psychosocial factors at work and musculoskeletal disease. Scand J Work Environ Health. 1993;19(5):297-312. PMID: 8296178

9. Jensen RC. Back injuries among nursing personnel related to exposure. Appl Occup Environ Hyg. 1990;5(1):38-45. DOI: 10.1080/1047322X.1990.10389586

10. Waters TR, Rockefeller K. Safe patient handling for rehabilitation professionals. Rehabil Nurs. 2010;35(5):216-22. PMID: 20836487

11. Vehmasvaara P. The physical load of emergency medical care and the development of tests to assess the physical prerequisites of the work ability of paramedics. A doctoral thesis Kuopio: Publications of the University of Kuopio. 2004;324.

12. Bureau of Labor Statistics US Department of Labor. Injury, Illnesses, and Fatalities: Frequently Asked Questions 2010 [updated 2011; cited 2011]. Available from: http://data.bls.gov/cgimbin/print.pl/iif/ oshfaq1.htm
13. Fujishiro K, Weaver JL, Heaney CA, Hamrick CA, Marras WS .The effect of ergonomic interventions in healthcare facilities on musculoskeletal disorders. Am J Ind Med. 2005;48(5):338-47. DOI: 10.1002/ajim.20225 PMID: 16254947

14. Hegarty C, Rosenberg J, Stecker R. Ergonomic evalution acute care nursing health. 2003.

15. Kim H, Dropkin J, Spaeth K, Smith F, Moline J. Patient handling and musculoskeletal disorders among hospital workers: Analysis of 7 years of institutional workers' compensation claims data. American journal of industrial medicine. 2012;55(8):683-90. DOI: $10.1002 /$ ajim.22006

16. Sulivan E, Decker P. Effective management in nursing. NewYork: Wesley; 1992

17. Karwowski W, S. Marras W. Editors Principles and Application in Engineering Series Occupational Ergonomics Engineering and Administrative Controls: Taylor \& Francis 2005.

18. Karhula K, Rönnholm T, Sjögren T, editors. Development of observation instrument for assessing work load on personnel involved in patient transfer tasks. NES 38th Annual Congress, NES Proceedings; 2006.

19. Smith DR, Sato M, Miyajima T, Mizutani T, Yamagata Z. Musculoskeletal disorders self-reported by female nursing students in central Japan: a complete cross-sectional survey. Int J Nurs Stud. 2003;40(7):725-9. PMID: 12965164

20. Sharifnia SH, Haghdoost AA, Hajihosseini F, Hoijati H. [Relationship between the musculoskeletal disorders with the ergonomic factors in nurses]Persian. Koomesh. 2011;12(4):372-8.

21. Scott PA. Global inequality, and the challenge for ergonomics to take a more dynamic role to redress the situation. Appl Ergon. 2008;39(4):495-9. DOI: 10.1016/j.apergo.2008.02.014 PMID: $\underline{18384753}$

22. Berry C. A guide to manual materials handling and back safety. USA: Commissioner of labor OSHA state plan designe; 2003.

23. Lagerström M, Josephson M, Pingel B, Tjernström G, Hagberg M. Evaluation of the implementation of an education and training programme for nursing personnel at a hospital in Sweden. Int J Ind Ergon. 1998;21(1):79-90. DOI: 10.1016/S0169-8141(97)00014-0 


\title{
Identification and Prioritization of the Factors Associated With Musculoskeletal Disorders in Nurses at a Specialized Hospital
}

\author{
Morteza GHolami ${ }^{1}$, Amir Kavousi ${ }^{2}$, Mahnaz Saremi ${ }^{2, *}$ \\ ${ }^{1}$ MSc Student, Department of Ergonomics, Faculty of Health, Safety and \\ Environment, Shahid Beheshti University of Medical Sciences, Tehran, Iran \\ ${ }^{2} \mathrm{PhD}$, Associate Professorof Biostatistics, Faculty of Health, Safety and Environment, \\ Shahid Beheshti University of Medical Sciences, Tehran, Iran \\ * Corresponding author: PhD, Assistant Professor of Ergonomics, Faculty of Health, \\ Safety and Environment, Shahid Beheshti University of Medical Sciences, Tehran, \\ Iran.E-mail:m.saremi@sbmu.ac.ir
}

DOI: $10.21859 /$ joe- 040416

Received: 10.02.2016

Accepted: 28.05 .2016

Keywords:

Musculoskeletal Disorders

Nurse

Patient Transfer

PTAI Index

\section{How to Cite this Article:} GHolami M, Kavousi A, Saremi M. Identification and Prioritization of the Factors Associated With Musculoskeletal Disorders in Nurses at a Specialized Hospital. J Ergo. 2016;4(2):44-51. DOI: $10.21859 /$ joe- 0402416

(C) 2016 Hamedan University of Medical Sciences.

\section{Abstract}

Introduction: Nursing in hospitals, because of the nature of the work, is a job in which workrelated musculoskeletal disorders are highly prevalent. The main cause of musculoskeletal disorder amongst nursing staff is the movement of patients. The aim of this study was to evaluate the risk of musculoskeletal disorders caused by movement of patients in nursing staff of a hospital affiliated with the Allah University of Medical Sciences.

Methods: This cross-sectional study was conducted on 325 nurses from 30 wards of Baqiyatallah hospital during summer 2015. Data were collected using demographic and Body map Musculoskeletal Disorders Questionnaires and PTAI index checklist. Statistical analysis was performed using the SPSS software version 16.

Results: Some musculoskeletal symptoms had been experienced during the last 12 months by \%78.3 of the nursing personnel. The results indicated that PTAI index score was significantly associated with musculoskeletal disorders occurrence $(\mathrm{P}<0.05)$

Conclusions: According to the findings, PTAI is an appropriate tool for musculoskeletal disorders risk identification and assessment due to patient handling in nursing personnel. 